\title{
Electrodynamics in Organic Dimer Insulators Close to Mott Critical Point
}

\author{
Marko Pinterić ${ }^{1,2}$, David Rivas Góngora ${ }^{1}$, Željko Rapljenović ${ }^{1}$, Tomislav Ivek ${ }^{1}$, Matija Čulo ${ }^{1}$, \\ Bojana Korin-Hamzić ${ }^{1}$, Ognjen Milat ${ }^{1}$, Branko Gumhalter ${ }^{1}$, Predrag Lazić ${ }^{3}$, \\ Miriam Sanz Alonso ${ }^{4}$, Weiwu Li ${ }^{4}$, Andrej Pustogow 4 (D), Guilherme Gorgen Lesseux ${ }^{4}$, \\ Martin Dressel ${ }^{4, * \text { (D) and Silvia Tomić }}{ }^{1, *}$ \\ 1 Institut za fiziku, Bijenička 46, HR-10000 Zagreb, Croatia; mpinter@ifs.hr (M.P.); drivas@ifs.hr (D.R.G.); \\ zrapljenovic@ifs.hr (Ž.R.); tivek@ifs.hr (T.I.); mculo@ifs.hr (M.Č.); bhamzic@ifs.hr (B.K.-H.); \\ milat@ifs.hr (O.M.); branko@ifs.hr (B.G.) \\ 2 University of Maribor, Faculty of Civil Engineering, Smetanova 17, SI-2000 Maribor, Slovenia \\ 3 Rudjer Bošković Institute, Bijenička 56, HR-10000 Zagreb, Croatia; plazicx@gmail.com \\ 4 1. Physikalisches Institut, Universität Stuttgart, Pfaffenwaldring 57, D-70550 Stuttgart, Germany; \\ weiwu.li@pi1.physik.uni-stuttgart.de (W.L.); andrej.pustogow@pi1.physik.uni-stuttgart.de (A.P.); \\ guilherme.gorgen-lesseux@pi1.physik.uni-stuttgart.de (G.G.L.) \\ * Correspondence: martin.dressel@pi1.physik.uni-stuttgart.de (M.D.); stomic@ifs.hr (S.T.)
}

Received: 26 March 2018 ; Accepted: 18 April 2018 ; Published: 27 April 2018

\begin{abstract}
Organic layered charge-transfer salts $\kappa$-(BEDT-TTF) ${ }_{2} X$ form highly frustrated lattices of molecular dimers in which strong correlations give rise to Mott insulating states situated close to the metal-to-insulator phase boundary. The salts $\kappa$-(BEDT-TTF $)_{2} \mathrm{Cu}_{2}(\mathrm{CN})_{3}$ and $\kappa$-(BEDT-TTF $)_{2} \mathrm{Ag}_{2}(\mathrm{CN})_{3}$ have been considered as prime candidates for a quantum spin liquid, while $\kappa$-(BEDT-TTF $)_{2} \mathrm{Cu}\left[\mathrm{N}(\mathrm{CN})_{2}\right] \mathrm{Cl}$ has been suggested as a prototypical charge-order-driven antiferromagnet. In this paper, we summarize and discuss several key results, including some not reported previously, obtained in search to clarify the competition of these two ground states. The origin of anomalous dielectric response found at low temperatures in all three salts is also discussed. We conclude by pointing out the relevant new insights into the role of frustration and random disorder in the suppression of magnetic ordering and formation of the spin liquid state.
\end{abstract}

Keywords: strongly correlated systems; mott insulators; quantum spin liquid; dielectric properties; vibrational properties; density functional theory

\section{Introduction}

Layered organic solids $\kappa$-(BEDT-TTF $)_{2} X\left(X=\mathrm{Cu}\left[\mathrm{N}(\mathrm{CN})_{2}\right] \mathrm{Cl}, \mathrm{Cu}_{2}(\mathrm{CN})_{3}\right.$ and $\left.\mathrm{Ag}_{2}(\mathrm{CN})_{3}\right)$ are systems in which strong correlations lead to Mott insulating states situated close to the metal-to-insulator phase boundary [1,2]. These systems are charge-transfer salts, i.e., they consist of conducting BEDT-TTF layers separated by non-conducting anion layers (Figure 1). Within BEDT-TTF layers, the triangular lattices of molecular dimers with one hole per dimer are formed with different degrees of frustration (Figure 2) [3-6]. Moderate pressure in the range of a few hundred bars up to $10 \mathrm{kbar}$ is sufficient to recover metallicity and superconducting ground state. At ambient pressure in these salts two different ground states can be established. Canted antiferromagnetic, i.e., weak ferromagnetic, state is formed in $\kappa$-(BEDT-TTF) ${ }_{2} \mathrm{Cu}\left[\mathrm{N}(\mathrm{CN})_{2}\right] \mathrm{Cl}$ (denoted as $\kappa-\mathrm{Cl}$ ) [7-9]. On the other hand, quantum spin liquid (QSL) develops in the two latter salts $\kappa$-(BEDT-TTF) ${ }_{2} X, X=\mathrm{Cu}_{2}(\mathrm{CN})_{3}$ and $\mathrm{Ag}_{2}(\mathrm{CN})_{3}$ (denoted as $\kappa-\mathrm{CuCN}$ and $\kappa-\mathrm{AgCN}$, respectively) [10-12]. QSL is commonly considered as a highly correlated fluctuating quantum spin state expected to appear in geometrically frustrated 
systems when quantum fluctuations are strong enough to preclude spin ordering down to zero temperature $[13,14]$. Historically, QSL was predicted theoretically as a resonating valence bond state to be the ground state of a perfect triangular lattice $S=1 / 2$ spin antiferromagnet instead of the conventional long-range antiferromagnetic (AF) order [15]. Experimentally, however, few real materials with triangular lattice, no spontaneously broken symmetry, and emergent fractional excitations have been discovered. Among them stand out the most recent discoveries of two novel QSL types. First is a high-temperature QSL in the layered transition metal dichalcogenide $1 \mathrm{~T}-\mathrm{TaS}_{2}$ [16] as a long-awaited prediction by Anderson. Second is a quantum liquid of magnetic and electric dipoles developed in a hydrogen-bonded molecular compound $\kappa-\mathrm{H}_{3}(\mathrm{Cat}-\mathrm{EDT}-\mathrm{TTF})_{2}$ [17].
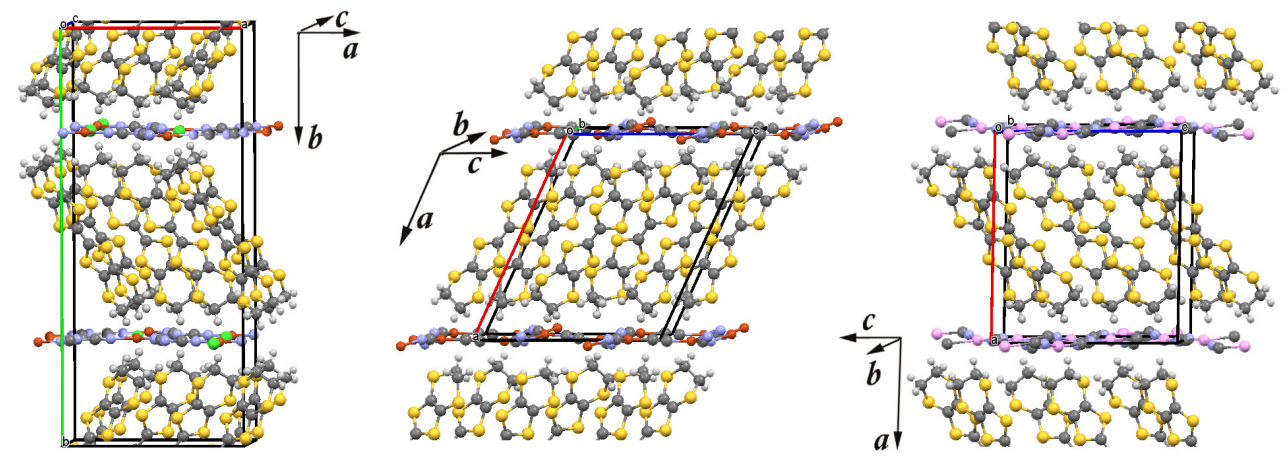

Figure 1. The crystal structure of the three layered organic charge-transfer salts. The lines mark the unit cell. Carbon, sulfur and hydrogen atoms of the BEDT-TTF molecule are colored in dark gray, yellow and light gray, respectively. In the anion network, chlorine, cooper, silver, carbon and nitrogen are colored in green, red, pink, dark grey and violet, respectively. (Left) Unit cell of $\kappa$-Cl. The space group is $P_{n m a}$. In each of two organic layers, related by mirror symmetry, all four BEDT-TTF molecules are equivalent. (Middle,Right) Extended unit cell of $\kappa-\mathrm{CuCN}$ and $\kappa-\mathrm{AgCN}$, respectively. The space group is commonly solved in $P 2{ }_{1} / c$ in which all four BEDT-TTF molecules are equivalent.
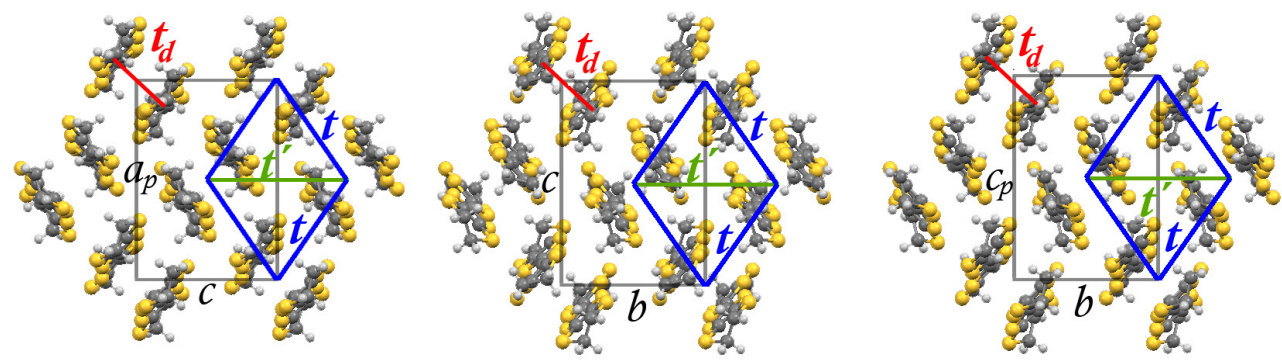

Figure 2. View of BEDT-TTF dimers arranged in anisotropic triangles in the three layered organic charge-transfer salts: (Left) $\kappa-\mathrm{Cl}$, projection of one of the layers is shown along the [110] direction; (Middle) $\kappa-\mathrm{CuCN}$, projected along the [100] direction; and (Right) $\kappa-\mathrm{AgCN}$, projected along the [10-1] direction. Neighboring dimers are rotated by $90^{\circ}$ with respect to each other. The interdimer transfer integrals are denoted by $t$ and $t^{\prime}$, and the intradimer transfer integral by $t_{\mathrm{d}}$. The ratio $t^{\prime} / t$ measures the degree of frustration. $t^{\prime} / t \approx 0.5$ indicates medium frustration for $\kappa-\mathrm{Cl}$, while $t^{\prime} / t \approx 0.85$ indicates high frustration for $\kappa-\mathrm{CuCN}$ and $\kappa-\mathrm{AgCN}$.

Common to all three $\kappa$-(BEDT-TTF $)_{2} X$ organic charge-transfer salts is an anomalous dielectric response [8,18-23], the origin of which remained elusive due to the missing experimental evidence of any sizeable electric dipoles on molecular dimers [19,23-25] proposed theoretically [26-34]. However, relying on the theory and based on the observation of Curie-like dielectric peak, hysteresis and time-dependent phenomena, a dipolar order has been proposed to set in $\kappa-\mathrm{Cl}$ acting as a driving force for the magnetic ground state [18]. Related open issue in this salt is that, instead of Curie-like peak, in the majority of measured single crystals, a relaxor response has been detected, suggesting 
that the dielectric response rather arises from the motion of charge domain walls between insulating ferromagnetic domains in the background impurity potential $[8,19]$. In two salts $\kappa-\mathrm{CuCN}$ and $\kappa-\mathrm{AgCN}$ with quantum spin liquid ground state, dielectric response is consistently found to be of relaxor type in all measured single crystals. The suppression of magnetic state has been mostly associated with highly frustrated molecular dimer lattices, but it has been argued that an additional mechanism, such as an exotic spin-dipolar coupling, is needed to induce quantum fluctuations which are strong enough to suppress magnetic order [26-34]. The reason for that is twofold. First is the proximity of Mott insulator-to-metal phase boundary where the charge degrees of freedom start to delocalize and thus a spin-only description cannot be sufficient. Second is the inability of frustrated triangular lattices to destroy the long-range magnetic order on their own [35-37]. The role of random disorder in this competition may also be important, as indicated by an experimental study on $\kappa$ - $\mathrm{Cl}$ showing that $\mathrm{X}$-rays suppress magnetic order and allow a spin liquid to form [38]. Finally, we have recently suggested that the non-conducting anions which contain frustration and disorder in the organic spin liquids may be a critical ingredient needed for the prevalence of quantum spin liquid over magnetic ordering [39].

In this paper, we address the issue of anomalous dielectric response and inherent randomness, and explore its origin and role in the competition between the AF and QSL ground state formation.

\section{Antiferromagnet with Ferroelectric Character $\kappa-(\mathrm{BEDT}-\mathrm{TTF})_{2} \mathrm{Cu}\left[\mathrm{N}(\mathrm{CN})_{2}\right] \mathrm{Cl}$}

At ambient pressure, $\kappa-\mathrm{Cl}$ system behaves as an insulator in dc limit in the whole temperature range, but, in the infrared range, a large temperature-dependent charge gap (Mott gap) develops only at temperatures lower than $50 \mathrm{~K}[19,40]$. The system was reported to show zero resistance and weak superconducting diamagnetic response at pressures as small as $100 \mathrm{bar}$, and a bulk superconductivity (SC) below $12.5 \mathrm{~K}$ at 300 bar [41-43]. On cooling, dc resistivity increases only weakly down to about $100 \mathrm{~K}$. This behavior is observed for majority of measured samples; the behavior of three single crystals is displayed in Figure 3, Inset in Left panel.

On further cooling below $100 \mathrm{~K}$, the dc resistivity increases steeply, indicating an insulator-toinsulator phase transition, right in the temperature range where the Mott gap develops. Interestingly, the rate by which dc resistivity rises seems to be much larger perpendicular to the dimer planes (i.e., along the $b$-axis) than within them $[18,44]$. Overall, dc resistivity data accumulated by us and the other authors however indicates that the phase transition temperature and the width of the transition extracted from the logarithmic resistivity derivative differ for different single crystals. Figure 3 shows distinct behaviors of the dc resistivity along the $b$-axis for three single crystals of $k$ - $\mathrm{Cl}$. The resistivity derivative of S2, as well as of the sample in Ref. [19] (see Figure 3 in [19]), shows a broad maximum centered at about $30 \mathrm{~K}$, indicative of the formation of a low-temperature phase at short-range scales. Conversely, samples S1 and S3 present a peak that is twice as narrow, higher, and positioned at a slightly lower temperature of $27 \mathrm{~K}$. However, the full width at half maximum is ten times larger than what is typical for the phase transition developed at the long-range scales, indicating a certain amount of disorder even in the crystals S1 and S3.

The nature of phase transition, based on dielectric data displaying a peak in the same temperature range (Figure 4, Right), was proposed to be ferroelectric and ascribed to the ordering of electric dipoles on BEDT-TTF dimers [18]. Moreover, it was argued that the dipolar ordering releases the frustration of dimers' spins on the triangular lattice and in this way drives the magnetic ordering which sets in at same temperatures. The exciting proposal of multiferroicity awaits for its final confirmation due to missing evidence for inversion symmetry breaking $[19,25]$. Thus, the nature of phase transitions detected in dc resistivity and dielectric response, and the origin of disorder in $\kappa-\mathrm{Cl}$ are still a mystery. We come back to this issue at the end of this section. An additional broad feature, situated at about $50 \mathrm{~K}$, is detected only for sample S3 (Figure 3, Right), right in the temperature range where the Mott gap opens.

Further, the most intriguing behavior is found for sample S2. Upon reducing temperature the resistivity rises with a much smaller rate, reaches maximum at about $12 \mathrm{~K}$ and falls down rapidly. 
Note that $12 \mathrm{~K}$ is the temperature below which bulk SC sets in at 300 bar. Notably, a similar drop of resistivity measured in dc limit, as well as at $35 \mathrm{GHz}$, has been reported before independently by Sushko et al. [45] and one of us [44], and attributed to SC islands growing within the insulating sample bulk. The suggestion has been supported by magnetization measurements indicating that the low temperature state of $\kappa-\mathrm{Cl}$ below critical pressure of 300 bar is an inhomogeneous mixture of SC and insulating magnetic phases [45]. Indeed, for sample S2, we do find evidence for the presence of SC domains at $T<T_{\mathrm{SC}} \approx 12 \mathrm{~K}$. These domains do not percolate down to $5 \mathrm{~K}$ since the resistivity remains finite (Figure 3). At these low temperatures only, a non-linear decrease of the low-frequency conductivity is found when increasing ac voltage (not shown) or applying magnetic field (Figure 5, Left). At zero magnetic field, there is a rise in conductivity below about $12 \mathrm{~K}$, as observed in dc resistivity measurements. Increasing the magnetic field shifts the SC phase transition to lower temperatures. For magnetic field of $5 \mathrm{~T}$ the SC phase transition is suppressed down to $5 \mathrm{~K}$.
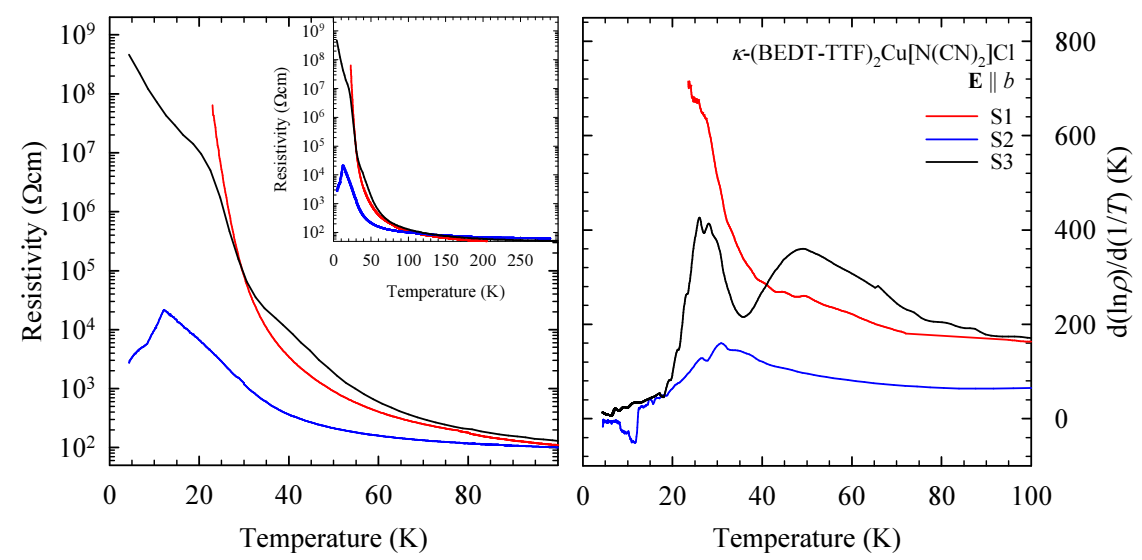

Figure 3. (Left) Distinct behaviors of the resistivity along the $b$ axis below $100 \mathrm{~K}$ for three single crystals of $\kappa-\mathrm{Cl}$ : S1 (red), S2 (blue) and S3 (black). Data for S3 are after [18]. The inset displays the resistivity behavior in the whole temperature range demonstrating a weak activated behavior similar for all three samples. (Right) Logarithmic resistivity derivative versus temperature.
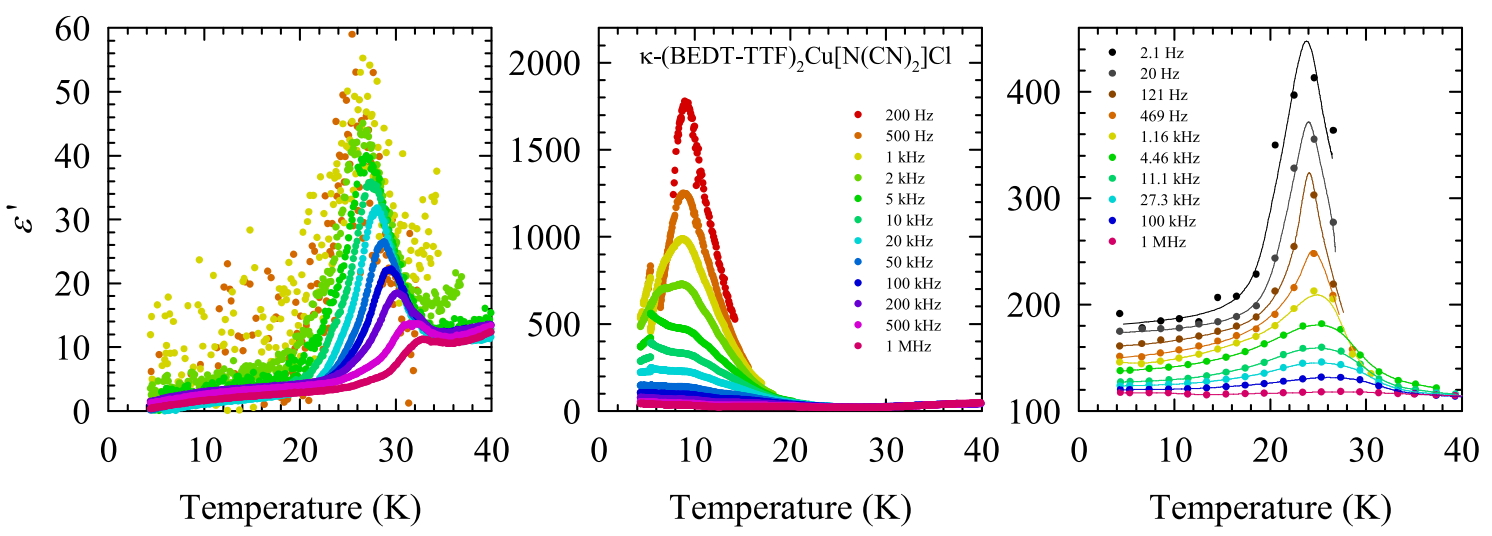

Figure 4. Real part of the dielectric function $\varepsilon^{\prime}$ as a function of temperature with the ac electric field applied E $\| b$ for three representative single crystals of $\kappa$-Cl: S1 (Left); S2 (Middle); and S3, after [18] (Right panel) The full lines in (Right) are guides for the eye.

Next, we address the dielectric function measured as a function of temperature and frequency along the $\mathbf{E} \| b$ i.e., perpendicular to the BEDT-TTF dimer layers. Figure 4 presents the real part of dielectric function $\varepsilon^{\prime}$ of three representative samples of $\kappa-\mathrm{Cl}$. Common to all of them is that below $40 \mathrm{~K}$, $\varepsilon^{\prime}$ features a pronounced peak, the high-temperature shoulders of which align to follow the same curve. Conversely, the peak's amplitudes and the positions in temperature strongly differ between them. 
The former seem to correlate with the magnitude of dc conductivity, but might also be influenced by different levels of disorder present in the three samples. The $\varepsilon^{\prime}$ peaks of sample S1 and S3 are situated at about $25 \mathrm{~K}$, showing a weak relaxor and a Curie-like behavior i.e., nearly frequency independent position of the peak, and reach similar values $\leq 100$ for frequencies $\geq 200 \mathrm{~Hz}$. A remarkably distinct result is observed for sample S2: the $\varepsilon^{\prime}$ peak is shifted to lower temperatures and centered at $T_{\mathrm{c}} \approx$ $9 \mathrm{~K}$ for all applied frequencies attaining a value as high as 1800 at $200 \mathrm{~Hz}$. Notably, $T_{\mathrm{c}}$ seems to coincide with $T_{\mathrm{SC}}$. Applied magnetic field reduces the onset of conductivity rise (Figure 5, Left) and concomitantly smooths the low-temperatures sides of the $\varepsilon^{\prime}$ peak and lowers its amplitude, the effect showing up only at temperatures below $T_{\mathrm{SC}} \approx 12 \mathrm{~K}$ (Figure 5, Right). Finally, the susceptibility behavior observed for sample S2 shows an increase, albeit rather broad, below about $22 \mathrm{~K}$ (Figure 6), as expected for the intra-layer antiferromagnetic ordering documented previously [7-9].

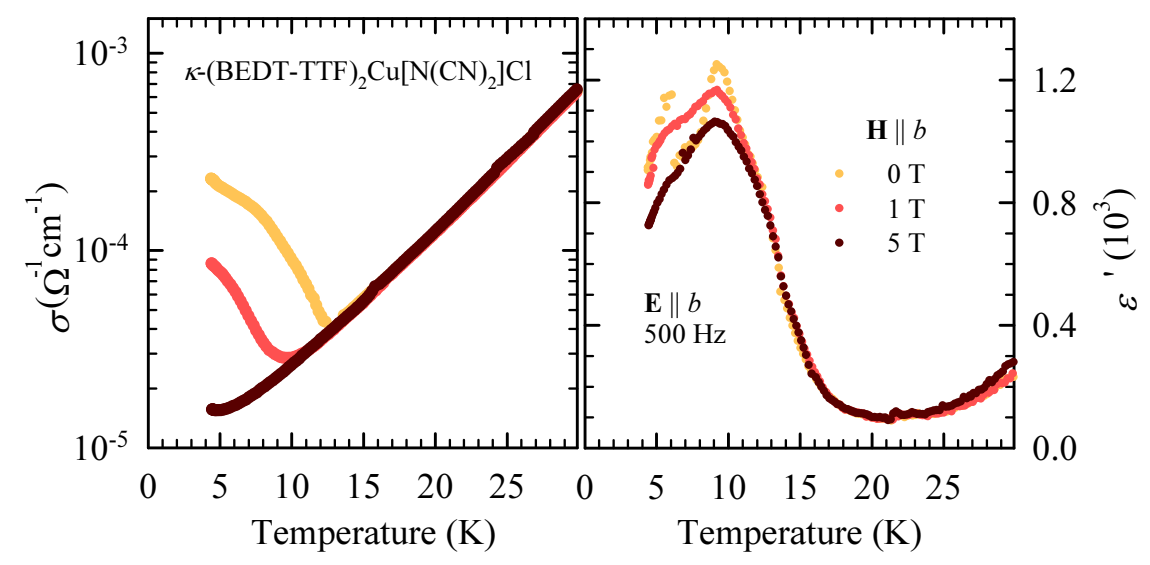

Figure 5. Magnetic field influence on sample S2 of $\kappa$-Cl. (Left) Conductivity $\sigma$ measured at $500 \mathrm{~Hz}$ for $\mathbf{E} \| b$ versus temperature measured at different magnetic field strengths $(\mathbf{H} \| b)$. At $\mathbf{H}=0 \mathrm{~T}$ there is a rise in conductivity below $12 \mathrm{~K}$, while the application of magnetic field shifts the onset of conductivity rise to lower temperatures, thus indicating a superconducting phase transition at $T_{\mathrm{SC}} \approx 12 \mathrm{~K}$. (Right) Real part of the dielectric function $\varepsilon^{\prime}$ as a function of temperature with the ac electric field applied $\mathbf{E} \| b$, and measured at different magnetic field strengths $(\mathbf{H} \| b)$. The effect of the magnetic field can be seen only below $12 \mathrm{~K}$. Increasing magnetic field lowers the peak amplitude.

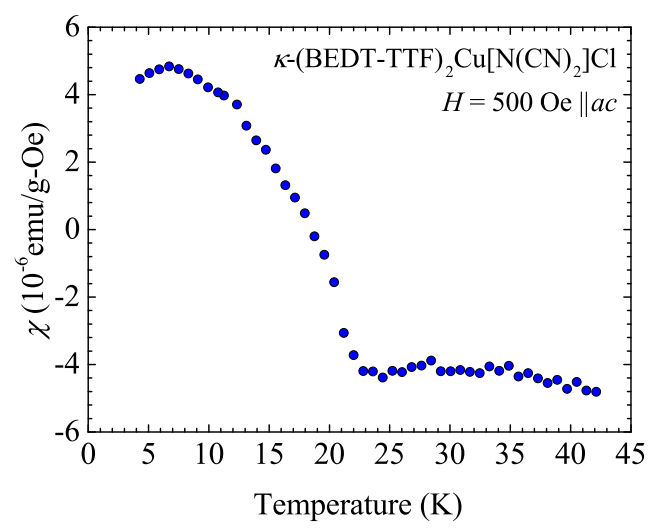

Figure 6. Susceptibility $\chi$ of sample $\mathrm{S} 2$ of $\kappa-\mathrm{Cl}$ as a function of temperature measured in a magnetic field of 500 Oe applied $\mathbf{H} \|$ ac-plane.

Importantly, for sample S2 both the magnetic phase transition and the insulator-to-insulator phase transition seem to be developed only at short-length scales, however they appear within the same temperature range as commonly observed in $\kappa$-Cl samples. On the other hand, the dielectric peak if taken as an indication of a ferroelectric-like ordering reveals itself as a feature separated from 
the phase transitions visible in dc transport and in susceptibility. Moreover, it is shifted to lower temperatures and appears concomitantly with the superconducting phase transition indicating the coexistence of ferroelectric-like order and SC. Thus, despite ferroelectricity being expected only in insulating systems, here it seems to coexist with dilute metallicity, indicating that ferroelectricity, together with magnetism, shares a common boundary with SC in the phase diagram. However, note that, while magnetic and SC are competing phases, the observed data suggest that ferroelectricity and SC support each other. Remarkably, Clay and Mazumdar recently developed a valence-bond theory of SC within which the SC state is reached by the destabilization of the molecular dimer order called paired-electron crystal (PEC) $[31,32,46]$. According to their theory, the low-temperature phase in sample S2 might be considered as consisting of segregated magnetic, PEC and SC phases. This outstanding result in organics bears similarities to the superconducting high- $T_{\mathrm{c}}$ cuprates, where the proximity of a charge-ordered state to the AF and the SC state has been established.

It is challenging to reconcile this exciting proposal with the missing experimental evidence of structural inversion symmetry breaking needed for formation of ferroelectricity. Namely, X-ray diffraction measurements have shown that the space group of $\kappa-\mathrm{Cl}$ is $P_{n m a}$ implying that all eight BEDT-TTF molecules and all four dimers in the unit cell are crystallographically equivalent [47]. Interestingly, NMR data reported in [9] seem to indicate four nonequivalent spin dimers sites present already at room temperature, but remarkably the nonequivalence disappeared once the magnetic state was established. The experimentally evidenced absence of any considerable electric dipoles on BEDT-TTF dimer sites [25] has promoted an alternative interpretation for the relaxor-like dielectric response observed in the most number of $\kappa-\mathrm{Cl}$ samples. This scenario, backed up by the generally observed tendency to phase segregation, invokes charged domain-walls between insulating ferromagnetic domains in the disordered background of the canted AF i.e., weak ferromagnetic phase $[8,19,22]$.

Here, we present vibrational spectroscopy data performed on sample S2 of $\kappa$-Cl. Figure 7 displays the most charge-sensitive intramolecular vibrational mode $v_{27}$ centered at $1469 \mathrm{~cm}^{-1}$ at $5 \mathrm{~K}$, which involves the anti-symmetric $\mathrm{C}=\mathrm{C}$ stretching of the rings within BEDT-TTF molecules, as a function of temperature [48-50]. The side band at $1473 \mathrm{~cm}^{-1}$ is present at all temperatures due to inequivalent lattice sites [25] and becomes visible at low temperatures as the modes become more narrow. The small satellite at $1478 \mathrm{~cm}^{-1}$ involves ethylene motions and is not related to the $v_{27}$ mode [50]. A possible charge inequality among the donor molecules can be quantitatively estimated from the well-known linear frequency-charge relation concluded by Yamamoto et al. [49]. The charge disproportionation can be directly determined from the frequency separation of the respective $v_{27}$ peaks via $2 \delta_{\rho}=\delta v_{27} /\left(140 \mathrm{~cm}^{-1} / e\right)$. In the case of $\kappa-\mathrm{Cl}$, however, no splitting of the mode occurs on lowering the temperature down to $5 \mathrm{~K}$, providing clear evidence that, even in sample S2 of $\kappa-\mathrm{Cl}$, no sizeable static charge redistribution exceeding the limit of $2 \delta_{\rho} \approx \pm 0.01 e$ takes place. The observation is in full accord with previous reports on $\kappa-\mathrm{Cl}[19,25]$. Given the estimated extremely small upper bound of charge imbalance and fast rate of charge oscillations within the dimer [25], such static or fluctuating electric dipoles associated with BEDT-TTF dimers can hardly explain the low-frequency dielectric response, in particular the one showing a sizeable Curie-like anomaly, as observed for sample S2 of $\kappa$-Cl. Importantly, in sample S2 the magnetic and dielectric anomaly, setting in at separated temperatures of $25 \mathrm{~K}$ and $9 \mathrm{~K}$, seem to suggest that it is a ferroelectricity which is driven by the magnetism, and not vice versa as proposed in [18].

Finally, origin of disorder in single crystals of $\kappa-\mathrm{Cl}$ is a very intriguing issue. The presence of disorder is suggested by the broadness of phase transition anomalies seen in dc transport and in susceptibility, as well as relaxor-like dielectric anomalies [51]. However, here the possibility that disorder originates in the anion layer, as suggested in the case of $\kappa-\mathrm{CuCN}$ and $\kappa-\mathrm{AgCN}[21,23,24]$, must be discarded since all cyanide $(\mathrm{CN})$ groups positioned between the copper $(\mathrm{Cu})$ atoms are ordered in a zigzag line along the $a$-axis so that the anion layer consists of one-dimensional chains (Figure 8). 
Alternatively, disorder may arise in the organic dimer layers due to the formation of segregated phases of similar energy [46]. This possibility should be explored further.

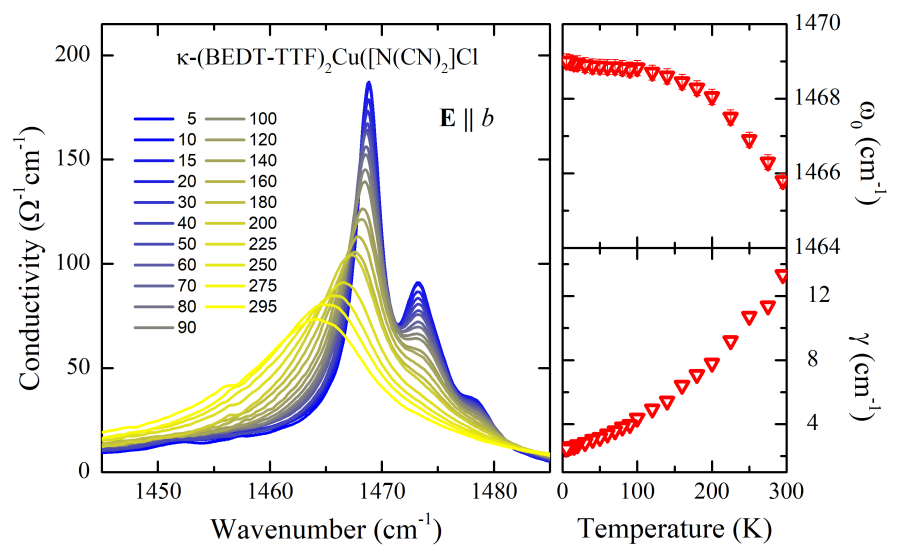

Figure 7. (Left) Temperature evolution of the intramolecular vibration $v_{27}$ in sample S2 of $\kappa$-Cl. (Right) Temperature dependence of the resonance frequency and damping obtained by Fano function fit of the mode.

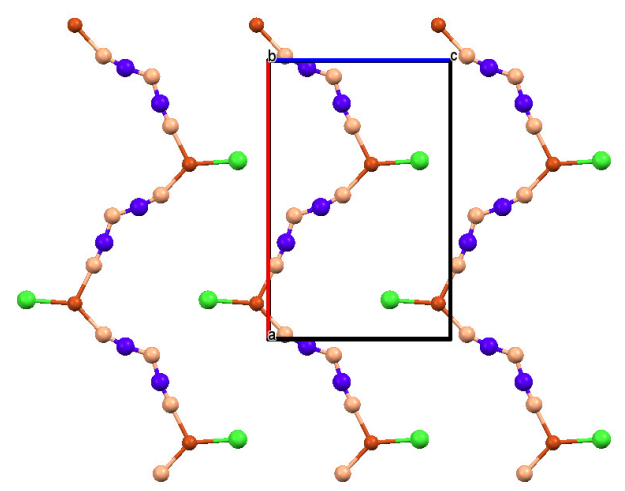

Figure 8. View of the anion layer of $\kappa-\mathrm{Cl}$ in the $a c$ plane projected along the $b$-axis. Copper is colored in red, and chlorine in green. Carbon and nitrogen are colored in blue and orange, respectively. The unit cell is marked as a rectangle.

\section{Quantum Spin Liquids in Disordered $\kappa$-(BEDT-TTF $)_{2} \mathrm{Cu}_{2}(\mathrm{CN})_{3}$ and $\kappa-(\mathrm{BEDT}-\mathrm{TTF})_{2} \mathrm{Ag}_{2}(\mathrm{CN})_{3}$}

$\kappa-\mathrm{CuCN}$ and $\kappa-\mathrm{AgCN}$ are Mott-Anderson insulators in which combined effects of strong Coulomb interaction and randomness have been well documented, experimentally and theoretically. dc charge transport within dimer planes happens via two-dimensional variable-range hopping in a wide low-temperature range. At elevated temperatures, it crosses-over to nearest-neighbor hopping $[21,23,52,53]$. Anomalously broad anisotropic dielectric response exhibits the relaxor-like ferroelectric behavior: with lower frequencies the magnitude of peak increases and its position shifts to lower temperature indicating a gradual freezing out [20,21,23]. Figure 9 displays the temperature-dependent real part of the dielectric function at selected frequencies along the $a^{*}$ direction where dimer and anion layers alternate. Notably, the out-of-plane response is one to three orders of magnitude weaker in strength, which correlates with the anisotropy of dc conductivity. Extensive study on different single crystals from different syntheses have revealed strong sample dependence of the dc transport and the dielectric response parameters [21]. Dielectric peak becomes smaller for larger disorder and the difference becomes more pronounced upon reducing temperatures. Similar effects due to disorder have been found previously in the dielectric 
response of the charge-density wave and ferroelectric charge ordered states in quasi-one-dimensional inorganic and organic compounds [22,54,55]. Disorder also affects the width of dielectric spectrum the broadening of which decreases implying a lower degree of cooperativity, the effect typically occurring in glassy systems [56]. Cooperativity appears well established within the dimer planes, while it develops gradually upon decreasing temperature along the out-of-plane direction [21,23]. In addition, for $\kappa-\mathrm{CuCN}$, sample-dependent dielectric and lattice anomalies were also detected in microwave [57] and thermal expansion measurements [58], respectively.

Dielectric response observed in $\kappa-\mathrm{CuCN}$ and $\kappa-\mathrm{AgCN}$ thus bears typical fingerprints of cooperative motion and glassy freezing in relaxor ferroelectrics [59]. Relaxor phenomenology assumes the existence of polar domains of low-symmetry and anticipates basically two mechanisms of dielectric response. It can be either due to the thermally activated reorientation of dipole moments within the domain, or to the motion of the interphase boundaries of the polar regions.

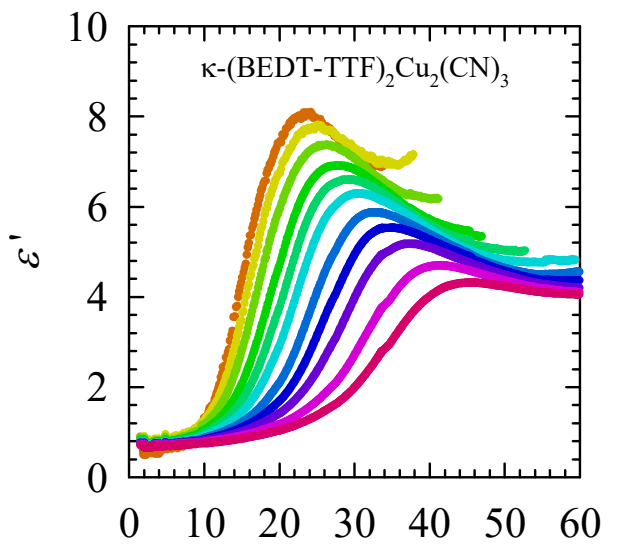

Temperature (K)

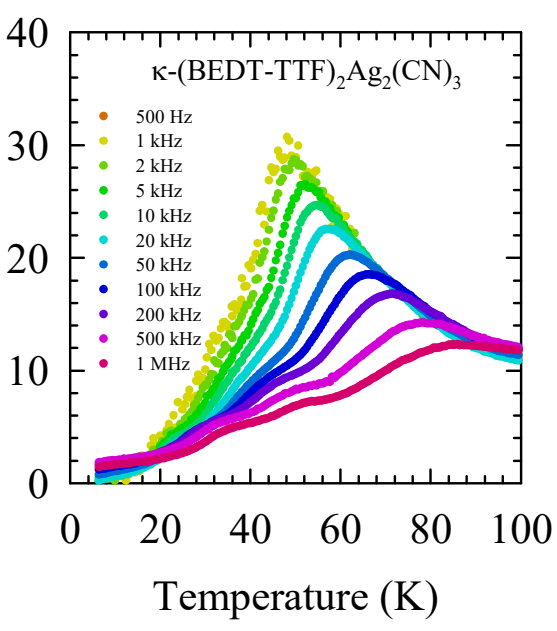

Temperature $(\mathrm{K})$

Figure 9. Real part of the dielectric function $\varepsilon^{\prime}$ as a function of temperature with the ac electric field applied $\mathbf{E} \| a^{*}$ for single crystals of: $\kappa-\mathrm{CuCN}$ (Left); and $\kappa$-AgCN (Right).

To examine if dipole moments emerge upon lowering the temperature in $\kappa-\mathrm{CuCN}$ and $\kappa-\mathrm{AgCN}$, vibrational spectroscopy was employed to probe the most charge-sensitive intramolecular vibrational mode $v_{27}$, which involves the anti-symmetric ring $\mathrm{C}=\mathrm{C}$ stretching of the BEDT-TTF molecule $[23,25]$. In Figures 10 and 11, the $v_{27}$ is shown for various temperatures in $\kappa-\mathrm{CuCN}$ and $\kappa-\mathrm{AgCN}$, respectively. In contrast to $\kappa-\mathrm{Cl}$, the side band at $1473 \mathrm{~cm}^{-1}$ is absent in different lattice symmetry; the satellite slightly below $1480 \mathrm{~cm}^{-1}$ is similar for all compounds discussed here. At $300 \mathrm{~K}$ the $v_{27}$ shows as a very strong peak centered at $1460 \mathrm{~cm}^{-1}$, as expected for half a hole per BEDT-TTF molecule. The mode is rather broad indicating the presence of fast charge fluctuations. Upon cooling no splitting of the mode occurs down to $10 \mathrm{~K}$, providing clear evidence that no sizeable static charge redistribution takes place in both $\kappa-\mathrm{CuCN}$ and $\kappa-\mathrm{AgCN}$. However, there is a slight difference. In $\kappa-\mathrm{CuCN}$, only one Fano mode was sufficient to describe the $v_{27}$ allowing only to conclude that there is no static charge imbalance larger than $2 \delta_{\rho} \approx \pm 0.01$ e (Figure 10). For $\kappa-\mathrm{AgCN}$, two Fano functions were required to fit the spectra, revealing the two maxima which indicate that two unequal crystallographic sites exist in the unit cell of $\kappa-\mathrm{AgCN}$ (Figure 11) [23]. With a linear shift of $140 \mathrm{~cm}^{-1}$ per unit charge, the charge imbalance $2 \delta_{\rho}$ is calculated by $2 \delta_{\rho}=\delta v_{27} /\left(140 \mathrm{~cm}^{-1} / e\right)$, where $\delta v_{27}$ is the difference in frequency positions between two maxima associated with two non-equal molecular or dimer sites. The two maxima are separated by $6 \mathrm{~cm}^{-1}$ which would correspond to an average charge imbalance of $2 \delta_{\rho} \approx \pm 0.05$.

Given that vibrational spectroscopy detects only a tiny charge imbalance and fast rate of oscillations, such dipoles cannot be invoked to explain the dielectric response at low frequencies. These fluctuating dipoles may show up in the microwave [57] and terahertz response [60]; however, the latter has been shown to involve coupled anion-dimer vibrations instead [24]. Alternatively, 
the collective motion of interphase boundaries of polar regions has to be considered as the pertinent mechanism in these systems. Therefore, the clue to understand relaxor properties of dielectric response lies in identifying the randomness and domain structure in $\kappa-\mathrm{CuCN}$ and $\kappa-\mathrm{AgCN}$. The structure of these salts is commonly solved within $P 2_{1} / c$ symmetry implying that all four molecules and two dimers within the unit cell are equivalent $[4,6,61]$. This means that any charge disproportionation between molecular and/or dimer sites is excluded.

However, randomness is revealed by X-ray diffraction data already at $300 \mathrm{~K}$ which show that, in addition to ordered $\mathrm{CN}$ groups (chain $\mathrm{CN}$ ) between $\mathrm{Cu} / \mathrm{Ag}$ atoms in the chains along the $b$-axis, there are $\mathrm{CN}$ groups (bridging $\mathrm{CN}$ ) located at the inversion centers. These bridging $\mathrm{CN}$ groups connect $\mathrm{Cu} / \mathrm{Ag}$ atoms along the $\mathrm{c}$-axis so that the anion network is formed in two-dimensions (Figure 12). The triangular coordination of $\mathrm{Cu}$ and $\mathrm{Ag}$ implies frustration since each $\mathrm{Cu} / \mathrm{Ag}$ atom can be linked either to two $\mathrm{N}$ and one $\mathrm{C}$ atom, or to one $\mathrm{N}$ atom and two $\mathrm{C}$ atoms introducing intrinsic disorder. This disorder is extended via hydrogen bonds connecting $\mathrm{CN}$ and ethylene groups onto the molecular dimers where it alters the charge distribution, and thus can cause anti-phase boundaries separating frustration-limited domains [21]. The perturbation of BEDT-TTF layers is expected to be stronger in $\kappa-\mathrm{CuCN}$, than in $\kappa-\mathrm{AgCN}$, since in the former all contacts between bridging $\mathrm{CN}$ and the terminal ethylene groups of the BEDT-TTF molecule are shorter than the sum of van der Waals radii [23]. Note that the disorder level in $\kappa-\mathrm{CuCN}$ and $\kappa-\mathrm{AgCN}$ represents an intrinsic property of these charge-transfer salts.

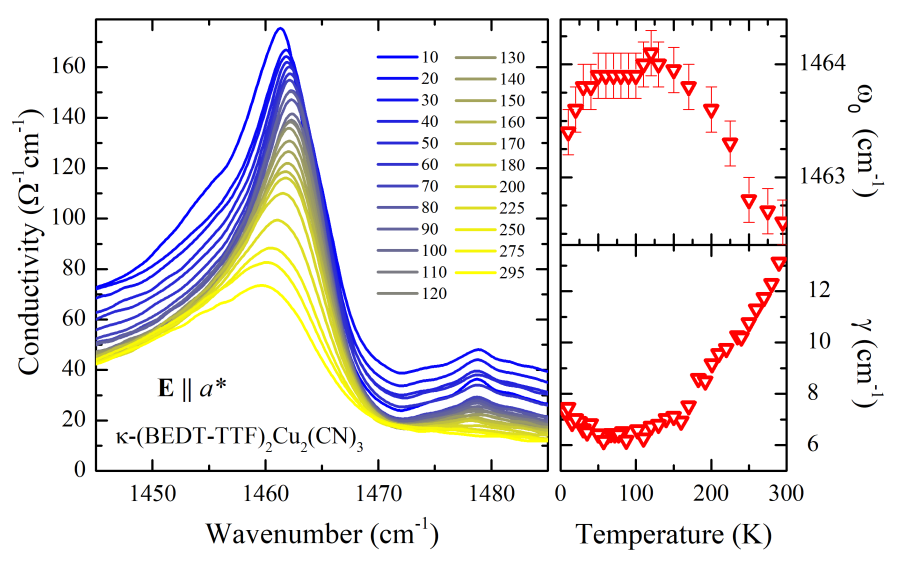

Figure 10. (Left) Temperature evolution of the intramolecular vibration $v_{27}$ of $\kappa$-CuCN. (Right) Temperature dependence of the resonance frequency and damping obtained by Fano function fit of the mode.

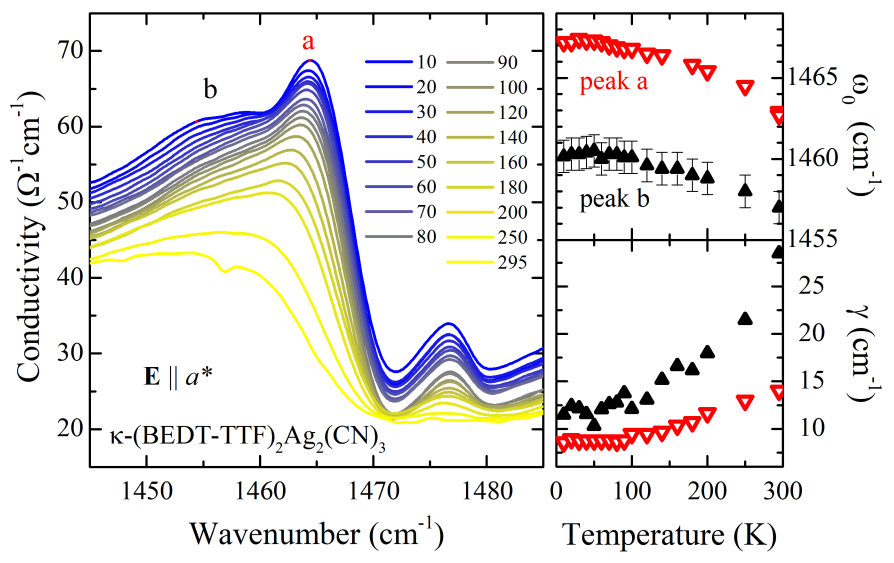

Figure 11. (Left) Temperature evolution of the intramolecular vibration $v_{27}$ of $\kappa$-AgCN. The two Fano functions required to fit the spectra indicate two crystallographically distinct sites. (Right) Temperature dependence of the resonance frequency and damping obtained by the fit using two Fano functions. 

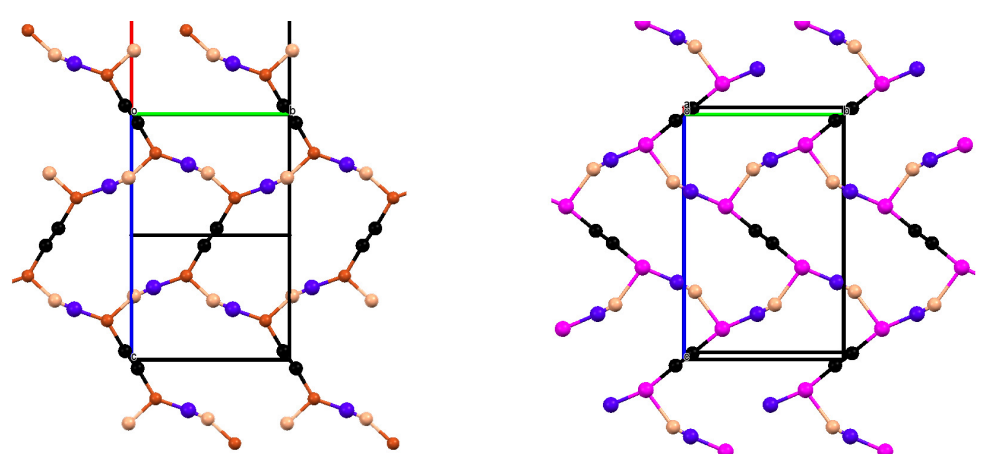

Figure 12. View of the anion network of $\kappa-\mathrm{CuCN}$ (Left) and $\kappa-\mathrm{AgCN}$ (Right) in the $b c$ plane projected along the $a^{*}$-axis. Cooper and silver are colored in red and violet, respectively. Carbon and nitrogen of ordered $\mathrm{CN}^{-}$groups are colored in blue and orange, while they are colored in black for $\mathrm{CN}^{-}$groups located at inversion centers. The unit cell is marked as a rectangle.

To get an insight as how such a disorder affects the electronic ground state, ab initio density functional theory (DFT) calculations have been carried out on $\kappa-\mathrm{CuCN}$ and $\kappa-\mathrm{AgCN}[23,24]$. Since two $\mathrm{CN}$ bridging groups in the unit cell occupy one of the two equally probable orientations, four different crystallographic configurations were constructed, based on experimental X-ray data, for $\kappa-\mathrm{CuCN}$ and $\kappa-\mathrm{AgCN}$. In each configuration, the entire system, with atomic positions and unit cell parameters, was allowed to relax resulting in electronic states with reduced symmetry as compared to $P 2_{1} / c$ and quasi-degenerate in energy; all states lie only $5-25 \mathrm{meV}$ above the relaxed state with minimum energy. Reduced symmetry implies that non-equivalent crystallographic sites (BEDT-TTF molecules and/or dimers) exist in the unit cell. In $\kappa-\mathrm{CuCN}$, the space group of all four states was reduced to $P 1$, while, in $\kappa-\mathrm{AgCN}$, the energy states were associated with the configurations of higher symmetry $P 2_{1}$ and $P_{c}$. These results indicate a higher level of disorder in $\kappa-\mathrm{CuCN}$ than in $\kappa-\mathrm{AgCN}$, which explains why it was possible to resolve two Fano modes in the $v_{27}$ mode in the $\kappa$-AgCN system.

Importantly, the quasi-degenerate electronic ground state implies a random domain structure in the real space of $\kappa-\mathrm{CuCN}$ and $\kappa-\mathrm{AgCN}$ at all temperatures. It thus supports the consideration of relaxor dielectric response explained above: low-energy excitations are caused by charged domain walls rather than by static or fluctuating electric dipoles. Upon cooling, screening gets gradually weaker due to the reduced number of charge carriers. This allows domains to be more "visible", the number of domain walls increases, and concomitantly the motion of domain walls become increasingly correlated. The detailed description of how and why the dielectric response evolve in temperature can be found in Refs. [21,23]. In closing, we note that the dielectric response scenario presented in this section, was recently backed up by the theoretical approach developed to explain anomalous dielectric response in strongly correlated molecular solids [62]. Most importantly, in-depth X-ray diffraction measurements of $\kappa-\mathrm{CuCN}$ in the wide temperature range and refinements, complemented by DFT calculations, have demonstrated that two nonequivalent dimers exist in the unit cell due to the reduced symmetry of the whole crystal [63].

\section{Van de Waals Interactions in $\kappa$-(BEDT-TTF $)_{2} X ; X=\mathrm{Cu}\left[\mathrm{N}(\mathrm{CN})_{2}\right] \mathrm{Cl}, \mathrm{Cu}_{2}(\mathrm{CN})_{3}$ and $\mathrm{Ag}_{2}(\mathrm{CN})_{3}$}

While there exists a general consent in the community of organic crystals on the importance of $\mathrm{vdW}$ interaction in their formation, its importance has never been clearly evidenced and quantified in the organic materials $\kappa$-(BEDT-TTF $)_{2} X$ with $X=\mathrm{Cu}\left[\mathrm{N}(\mathrm{CN})_{2}\right] \mathrm{Cl}_{1} \mathrm{Cu}_{2}(\mathrm{CN})_{3}$ and $\mathrm{Ag}_{2}(\mathrm{CN})_{3}$. These three kappa-BEDT-TTF materials, as well as other BEDT-TTF compounds are not simple molecular crystals, rather they should be classified as complex chemically bound charge transfer or cation-anion bulk solids. The previous DFT studies on different organic charge-transfer salts have neither included nor discussed the van der Waals ( $\mathrm{vdW}$ ) interactions at all [64-70]. The only exception is found in our work $[23,24,39]$. 
In the present DFT calculations, the experimental structure obtained from X-ray diffraction measurements at $100 \mathrm{~K}, 150 \mathrm{~K}$ and $100 \mathrm{~K}$ was used for the atomic coordinates of $\kappa-\mathrm{CuCN}, \kappa-\mathrm{AgCN}$ and $\kappa-\mathrm{Cl}$, respectively $[23,24,47]$. Two types of DFT functionals have been used: the standard semilocal PBE functional and nonlocal vdW-DF functional. The structure was relaxed to allow for the change of volume and shape of the unit cell together with the atomic positions. The parameters of the resulting relaxed state structures with minimum energy are displayed in Table $1(\kappa-\mathrm{CuCN})$, Table $2(\kappa-\mathrm{AgCN})$ and Table $3(\kappa-\mathrm{Cl})$.

Table 1. Unit cell parameters of $\kappa-\mathrm{CuCN}$ obtained from X-ray diffraction measurements at $100 \mathrm{~K}$ (left column), ab initio calculations based solely on PBE functional (central column) and on vdW-DF functional (right column). Relative deviations from experimental values are given in parentheses.

\begin{tabular}{cccc}
\hline Unit Cell Parameters & Exp & Calc:PBE & Calc:vdW-DF \\
\hline$a$ & $15.9644 \AA$ & $16.9706 \AA(+6.3 \%)$ & $16.1809 \AA(+1.4 \%)$ \\
$b$ & $8.5618 \AA$ & $8.75745 \AA(+2.3 \%)$ & $8.62586 \AA(+0.8 \%)$ \\
$c$ & $13.2662 \AA$ & $13.6497 \AA(+2.9 \%)$ & $13.3523 \AA(+0.7 \%)$ \\
$\alpha$ & $90.000^{\circ}$ & $89.9968^{\circ}$ & $90.0037^{\circ}$ \\
$\beta$ & $114.067^{\circ}$ & $116.409^{\circ}$ & $114.088^{\circ}$ \\
$\gamma$ & $90.000^{\circ}$ & $90.0037^{\circ}$ & $89.9994^{\circ}$ \\
$V$ & $1655.65 \AA^{3}$ & $1816.90 \AA^{3}(+9.7 \%)$ & $1701.35 \AA^{3}(+2.8 \%)$ \\
\hline
\end{tabular}

Table 2. Unit cell parameters of $\kappa$-AgCN obtained from X-ray diffraction measurements at $150 \mathrm{~K}$ (left column), ab initio calculations based solely on PBE functional (central column) and on vdW-DF functional (right column). Relative deviations from experimental values are given in parentheses.

\begin{tabular}{cccc}
\hline Unit Cell Parameters & Exp & Calc:PBE & Calc:vdW-DF \\
\hline$a$ & $14.969900 \AA$ & $15.6693 \AA(+4.7 \%)$ & $15.1161 \AA(+0.98 \%)$ \\
$b$ & $8.656500 \AA$ & $9.04653 \AA(+4.5 \%)$ & $8.72513 \AA(+0.79 \%)$ \\
$c$ & $13.216900 \AA$ & $13.8238 \AA(+4.6 \%)$ & $13.3487 \AA(+1.0 \%)$ \\
$\alpha$ & $90.000000^{\circ}$ & $90.0000^{\circ}$ & $90.0000^{\circ}$ \\
$\beta$ & $91.389000^{\circ}$ & $94.6823^{\circ}$ & $91.3952^{\circ}$ \\
$\gamma$ & $90.000000^{\circ}$ & $90.0000^{\circ}$ & $90.0000^{\circ}$ \\
$V$ & $1712.23 \AA^{3}$ & $1953.03 \AA^{3}(+14.1 \%)$ & $1760.03 \AA^{3}(+2.8 \%)$ \\
\hline
\end{tabular}

Table 3. Unit cell parameters of $\kappa-\mathrm{Cl}$ obtained from $\mathrm{X}$-ray diffraction measurements at $100 \mathrm{~K}$ (left column), ab initio calculations based solely on PBE functional (central column) and on vdW-DF functional (right column). Relative deviations from experimental values are given in parentheses.

\begin{tabular}{cccc}
\hline Unit Cell Parameters & Exp & Calc:PBE & Calc:vdW-DF \\
\hline$a$ & $12.885200 \AA$ & $12.9769 \AA(+0.7 \%)$ & $12.8334 \AA(-0.4 \%)$ \\
$b$ & $29.575899 \AA$ & $29.6637 \AA(+0.3 \%)$ & $29.5323 \AA(-0.2 \%)$ \\
$c$ & $8.416100 \AA$ & $8.45028 \AA(+0.4 \%)$ & $8.23316 \AA(-2.2 \%)$ \\
$\alpha$ & $90.000000^{\circ}$ & $90.0000^{\circ}$ & $90.0000^{\circ}$ \\
$\beta$ & $90.000000^{\circ}$ & $90.0000^{\circ}$ & $90.0000^{\circ}$ \\
$\gamma$ & $90.000000^{\circ}$ & $90.0000^{\circ}$ & $90.0000^{\circ}$ \\
$V$ & $3207.303 \AA^{3}$ & $3252.875 \AA^{3}(+1.4 \%)$ & $3120.366 \AA^{3}(-2.7 \%)$ \\
\hline
\end{tabular}

The inadequacy of DFT calculations without the vdW interactions is found in $\kappa-\mathrm{CuCN}$ and $\kappa-\mathrm{AgCN}$ for which the absence of $\mathrm{vdW}$ interaction produces deviations of the computed unit cell parameters from the experimental ones at the level of $10 \%$ and $14 \%$, respectively (Tables 1 and 2). Hence, only the DFT calculations with the non-local vdW-DF functional can clarify the impact of disorder on the electronic ground state (see Section 3). Intriguingly enough, the vdW forces are almost irrelevant in $\kappa-\mathrm{Cl}$ (Table 3) thereby indicating that the latter structure is dominantly determined by the one-dimensional chains of anion $\mathrm{Cu}\left[\mathrm{N}(\mathrm{CN})_{2}\right] \mathrm{Cl}$ chemically bonded to the organic dimers. 


\section{Materials and Methods}

All measurements were performed on high-quality single crystals grown by J. A. Schlueter, Argonne $\left(\kappa-(\mathrm{BEDT}-\mathrm{TTF})_{2} \mathrm{Cu}\left[\mathrm{N}(\mathrm{CN})_{2}\right] \mathrm{Cl}\right) ; \mathrm{K}$. Miyagawa and K. Kanoda, Tokyo University $\left(\kappa-(\mathrm{BEDT}-\mathrm{TTF})_{2} \mathrm{Cu}_{2}(\mathrm{CN})_{3}\right)$; and T. Hiramatsu, Y. Yoshida and G. Saito, Meijo University $\left.(\kappa \text {-(BEDT-TTF })_{2} \mathrm{Ag}_{2}(\mathrm{CN})_{3}\right)$. Sample S1 of $\kappa-\mathrm{Cl}$ was of cube-like shape with an area of $0.4 \times 0.5 \mathrm{~mm}^{2}$, and a thickness of $0.6 \mathrm{~mm}$. Sample S2 of $\kappa-\mathrm{Cl}$ was plate-like with an $a c$-plane area of $0.8 \times 0.9 \mathrm{~mm}^{2}$. and a thickness of $0.4 \mathrm{~mm}$. Contacts for dc and dielectric measurements along the out-of-plane direction were made by applying conductive carbon paint directly to the surface of the sample [21,23]. DC resistivity was measured by a standard four contact technique. Temperature-dependent dielectric measurements were performed from room temperature down to $4.2 \mathrm{~K}$ (crystals S1 and S2 of $\kappa-\mathrm{Cl}$ ) and $1.8 \mathrm{~K}$ (crystal of $\kappa-\mathrm{CuCN}$ ) in the frequency range $40 \mathrm{~Hz}-10 \mathrm{MHz}$ using Agilent $4294 \mathrm{~A}$ and Keysight E4980AL-102 precision impedance analyzers. Care was taken to subtract the background capacitance of the set-up and sample holder, as well as to exclude any possible extrinsic effects due to sample preparation. Magnetic-field-dependent dielectric measurements of sample S2 of $\kappa$-Cl were performed in the magnetic field up to $5 \mathrm{~T}$ aligned along the out-of-plane crystallographic axis. Vibrational spectroscopy measurements were made on the thin side of the crystals by an IR microscope attached to a Bruker Vertex 80v Fourier-transform spectrometer with $1 \mathrm{~cm}^{-1}$ resolution. Magnetic susceptibility of sample $\mathrm{S} 2$ of $\kappa$ - $\mathrm{Cl}$ was performed by using a superconducting quantum interference device (SQUID) magnetometer and the data were corrected for the contribution of sample holder.

First principles calculations of the electronic structure was carried out by using the self-consistently implemented nonlocal van der Waals density functional (vdW-DF) [71-73] for correlation with optB88 for exchange [74]. The expansion in the plane waves was done with a cutoff energy of $700 \mathrm{eV}$. The Brillouin zone was sampled by $1 \times 2 \times 2$ Monkhorst-Pack choice of $k$-points [75]. For the atomic coordinates, the experimental structure obtained by $\mathrm{X}$-ray diffraction measurements was used. The structure was relaxed allowing for change of the volume and the shape of the unit cell together with atomic positions within, until the forces on atoms dropped below $1 \mathrm{meV} / \AA$.

To ensure the chemical composition and exclude presence of any bromine in sample S2 of $\kappa-\mathrm{Cl}$, we examined the crystal symmetry and composition [76]. The X-ray data were collected at $300 \mathrm{~K}$ with graphite monochromated Mo-K $\alpha$ radiation by $\omega$-scans on an Oxford Diffraction KM4 XCALIBUR2 CCD diffractometer. Data acquisition and reduction was performed using the CrysAlisPro software package. The structure was solved by direct methods using SHELXS and the refinement was performed using SHELXL [77]. Energy Dispersive X-ray Spectra (EDXS) of sample S2 of $\kappa$-Cl, were acquired using Bruker detector within Tescan Vega III Easyprobe Scanning Electron Microscope (SEM).

Figure 13 shows the result of structural refinements of X-ray data within models containing either $\mathrm{Cl}$ (Figure 13, Left) or Br (Figure 13, Right). All atoms are drawn with 50\% probability ellipsoids of thermal displacement. Importantly, the size of ellipsoids is convincingly small for $\mathrm{Cl}$, while their size is unrealistically inflated if the presence of $\mathrm{Br}$ is assumed. In addition, the distance of $2.22 \AA$ between $\mathrm{Cu}$ and neighboring $\mathrm{Cl}$ atom (represented by lines) is close to $2.26 \AA$ which is typical for $\mathrm{Cu}-\mathrm{Cl}$ bond, thus confirming the presence of $\mathrm{Cl}$ in the structure. Finally, the presence of $\mathrm{Br}$ larger than a few percent can be rejected since the corresponding bond in the case of Br would be longer and amount to $2.42 \AA$. Therefore, overall results of the structural refinements strongly indicate that the measured single crystal is $\kappa-\mathrm{Cl}$ and not $\kappa-\mathrm{Br}$.

Figure 14 shows EDXS recorder at $300 \mathrm{~K}$. Characteristic lines for C, N, S and Cu were observed with intensities that correspond to the $\kappa-\mathrm{Cl}$ material. On the other hand, no significant intensity of $\mathrm{Br}$ lines, expected if trace amount of $\mathrm{Br}$ is present, were observed. 

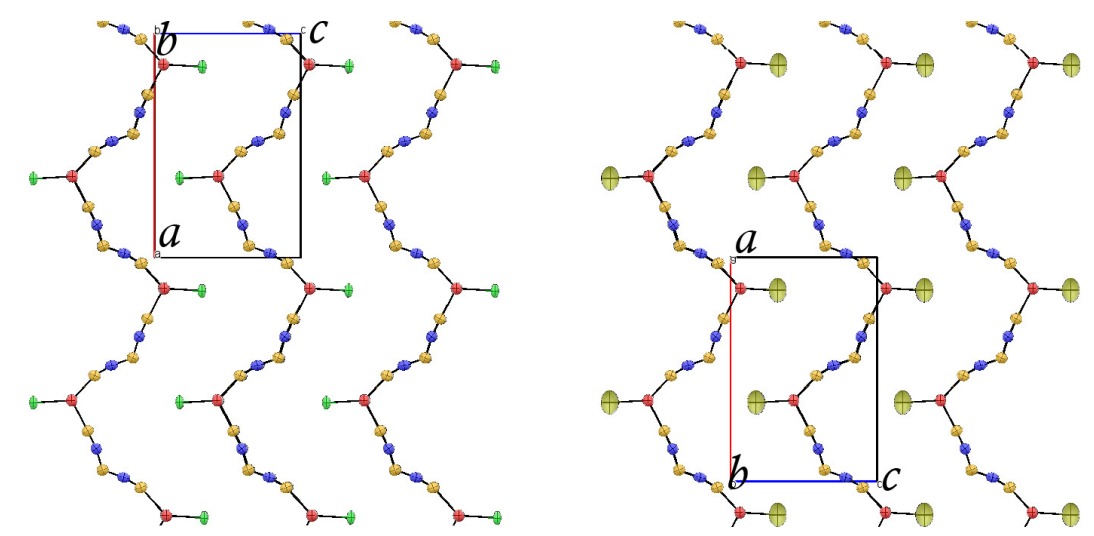

Figure 13. Perspective view of zigzag polymeric anion chains as obtained by structural refinements of X-ray data assuming either: $\kappa-\mathrm{Cl}$ (Left); or $\kappa$ - $\mathrm{Br}$ (Right). View shows the ac plane. Copper, carbon and nitrogen are shown in red, blue and orange, respectively. Chlorine and bromine ligands on each copper atom are colored in dark and light green, respectively. All atoms are drawn with $50 \%$ probability ellipsoids of anisotropic thermal displacement parameters. Unit cell is marked as a rectangle.

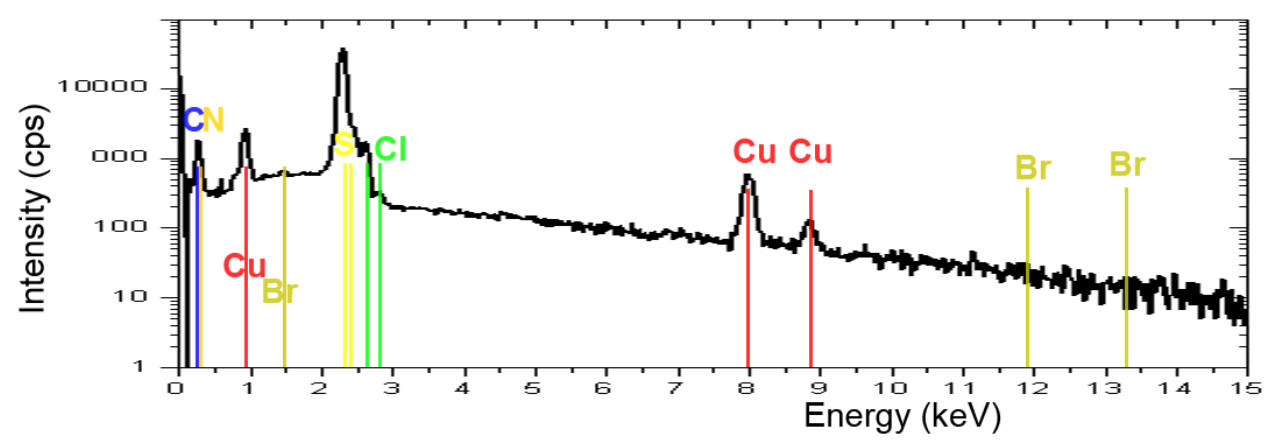

Figure 14. Energy Dispersive X-ray Spectra (EDXS) recorded at $300 \mathrm{~K}$. Only characteristic lines for $\mathrm{C}, \mathrm{N}, \mathrm{S}$ and $\mathrm{Cu}$, and in particular for $\mathrm{ClK} \alpha$ at $2.59 \mathrm{keV}$ and for $\mathrm{ClK} \beta$ at $2.83 \mathrm{keV}$ were observed with intensities that correspond to the chemical formula of $\kappa-\mathrm{Cl}$. On the other hand, no significant intensity for lines $\operatorname{BrK} \alpha 1$ and $\operatorname{BrK} \alpha 2$ at $11.92 \mathrm{keV}$, for $\operatorname{BrK} \beta$ at $13.29 \mathrm{keV}$, and for $\operatorname{BrL} \alpha$ at $1.49 \mathrm{keV}$ were observed, which would be expected in the presence of $\mathrm{Br}$.

\section{Conclusions}

Mott insulators $\kappa-\mathrm{CuCN}$ and $\kappa-\mathrm{AgCN}$ with the quantum spin liquid ground state exhibit high geometrical frustration in the organic dimers and in the non-conducting anions. Frustration in the anions arises as a combined effect due to the triangular coordination of copper/silver atoms and the bridging cyanide groups sitting in the inversion centers. The resulting disorder is mapped via anion-cation coupling onto the organic dimers. It gives rise to charge redistribution and a ground state which consists of several quasi-degenerate electronic states with reduced symmetry.

Remarkably, QSL is replaced by the antiferromagnetic ground state in $\kappa-\mathrm{Cl}$ where disorder due to inherent randomness in the anions and related symmetry reduction are absent. This result strongly suggests that the inherent randomness may be of a crucial importance for the prevalence of QSL over the antiferromagnetic ordering. In addition, van der Waals forces are found to contribute to inter-dimer interactions and the ground state structure in $\kappa-\mathrm{CuCN}$ and $\kappa-\mathrm{AgCN}$, whereas their role appears insignificant in $\kappa-\mathrm{Cl}$ where chemical bonding dominantly determines the inter-dimer interactions besides the cation-anion coupling.

Anomalous dielectric peak shows up as an ubiquitous property independent on the nature of the ground state in the spin sector. It is now well established that, in $\kappa-\mathrm{CuCN}$ and $\kappa-\mathrm{AgCN}$, this arises due to the cooperative motion of charged domain walls. On the other hand, in $\kappa-\mathrm{Cl}$, some mysteries remain 
unresolved. The charged domain wall motion might also be in action in $\kappa-\mathrm{Cl}$ if the tendency to phase segregation is invoked due to canted spins in the antiferromagnetic state. Further experimental efforts are vital in search for structural inversion symmetry breaking in order to clarify the ferroelectricity in $\kappa-\mathrm{Cl}$. The possibility that ferroelectricity is proximate to the magnetic and superconducting phases is most exciting and deserves more attention in future studies. Experiments such as X-ray diffraction and dielectric spectroscopy under pressure, on irradiated samples and in magnetic field are envisaged as tools to elucidate respective roles of correlations, randomness and charge-spin coupling in the phase diagram.

Author Contributions: DC and dielectric measurements and the data analysis were performed by Marko Pinteric, David Rivas Gongora, and Zeljko Rapljenovic, with help of Tomislav Ivek and Matija Culo. Dielectric measurements in magnetic field were designed by Tomislav Ivek and Matija Culo. Structural data analysis was performed by Ognjen Milat. DFT calculations were performed by Predrag Lazic. Vibrational experiments and data analysis were performed by Miriam Sanz Alonso, Andrej Pustogow, and Weiwu Li. Susceptibility experiments and data analysis were performed by Guilherme Gorgen Lesseux. The interpretation of the data and the text of the manuscript were drafted by Silvia Tomic and Tomislav Ivek. All authors contributed to the discussion and the final manuscript.

Acknowledgments: X-ray diffraction measurements by V. Stilinović and SEM measurements by J. Macan are gratefully acknowledged. We would like to thank V. Dobrosavljević, E. Canadell, R. T. Clay, P. Foury, V. Ilakovac, S. Mazumdar and J-P. Pouget for many enlightening discussions. This work has been supported by the Croatian Science Foundation project IP-2013-11-1011 and by the Deutsche Forschungsgemeinschaft (DFG).

Conflicts of Interest: The authors declare no conflict of interest.

\section{References}

1. Kanoda, K.; Kato, R. Mott Physics in Organic Conductors with Triangular Lattices. Annu. Rev. Condens. Matter Phys. 2011, 2, 167. [CrossRef]

2. Powell, B.J.; McKenzie, R.H. Quantum frustration in organic Mott insulators: From spin liquids to unconventional superconductors. Rep. Prog. Phys. 2011, 74, 056501. [CrossRef]

3. Kandpal, H.C.; Opahle, I.; Zhang, Y.-Z.; Jeschke, H.O.; Valentí, R. Revision of Model Parameters for $\kappa$-Type Charge Transfer Salts: An Ab Initio Study. Phys. Rev. Lett. 2009, 103, 067004. [CrossRef] [PubMed]

4. Jeschke, H.O.; de Souza, M.; Valentí, R.; Manna, R.S.; Lang, M.; Schlueter, J.A. Temperature dependence of structural and electronic properties of the spin-liquid candidate $\kappa-(\mathrm{BEDT}-\mathrm{TTF})_{2} \mathrm{Cu}_{2}(\mathrm{CN})_{3}$. Phys. Rev. B 2012, 85, 035125. [CrossRef]

5. Koretsune, T.; Hotta, C. Evaluating model parameters of the $\kappa$ and $\beta^{\prime}$-type Mott insulating organic solids. Phys. Rev. B 2014, 89, 045102. [CrossRef]

6. Hiramatsu, T.; Yoshida, Y.; Saito, G.; Otsuka, A.; Yamochi, H.; Maesato, M.; Shimizu, Y.; Ito, H.; Nakamura, Y.; Kishida, $\mathrm{H}$.; et al. Design and preparation of a new quantum spin liquid candidate, $\kappa$ - $(\mathrm{ET})_{2} \mathrm{Ag}_{2}(\mathrm{CN})_{3}$, having a nearby superconductivity. Bull. Chem. Soc. Jpn. 2017, 90, 1073-1082. [CrossRef]

7. Miyagawa, K.; Kawamoto, A.; Nakazawa, Y.; Kanoda, K. Antiferromagnetic Ordering and Spin Structure in the Organic Conductor, $k$-(BEDT-TTF $)_{2} \mathrm{Cu}\left[\mathrm{N}(\mathrm{CN})_{2}\right] \mathrm{Cl}$. Phys. Rev. Lett. 1995, 75, 1174. [CrossRef] [PubMed]

8. Pinterić, M.; Miljak, M.; Biškup, N.; Milat, O.; Aviani, I.; Tomić, S.; Schweitzer, D.; Strunz, W.; Heinen, I. Magnetic anisotropy and low frequency dielectric response of weak ferromagnetic phase in $\kappa$-(BEDT-TTF $)_{2} \mathrm{Cu}\left[\mathrm{N}(\mathrm{CN})_{2}\right] \mathrm{Cl}$. Eur. Phys. J. B 1999, 11, 217.

9. Smith, D.F.; De Soto, S.M.; Slichter, C.P. Dzialoshinskii-Moriya interaction in the organic superconductor $\kappa$-(BEDT-TTF $)_{2} \mathrm{Cu}\left[\mathrm{N}(\mathrm{CN})_{2}\right] \mathrm{Cl}$. Phys. Rev. B 2003, 68, 024512. [CrossRef]

10. Shimizu, Y.; Miyagawa, K.; Kanoda, K.; Maesato, M.; Saito, G. Spin liquid state in an organic Mott insulator with a triangular lattice. Phys. Rev. Lett. 2003, 91, 107001. [CrossRef] [PubMed]

11. Shimizu, Y.; Hiramatsu, T.; Maesato, M.; Otsuka, A.; Yamochi, H.; Ono, A.; Itoh, M.; Yoshida, M.; Takigawa, M.; Yoshida, Y.; et al. Pressure-tuned exchange coupling of a quantum spin liquid in the molecular triangular lattice $\kappa$-(BEDT-TTF $)_{2} \mathrm{Ag}_{2}(\mathrm{CN})_{3}$. Phys. Rev. Lett. 2016, 117, 107203. [CrossRef] [PubMed]

12. Zhou, Y.; Kanoda, K.; Ng, T.-K. Quantum spin liquid states. Rev. Mod. Phys. 2017, 89, 025003. [CrossRef]

13. Balents, L. Spin liquids in frustrated magnets. Nature 2010, 464, 199. [CrossRef] [PubMed]

14. Savary, L.; Balents, L. Quantum spin liquids: A review. Rep. Prog. Phys. 2017, 80, 016502. [CrossRef] [PubMed] 
15. Anderson, W.P. Resonating valence bonds: A new kind of insulator? Mat. Res. Bull. 1973, 8, $153-160$. [CrossRef]

16. Klanjšek, M.; Zorko, A.; Žitko, R.; Mravlje, J.; Jagličić, Z.; Kumar Biswas, P.; Prelovšek, P.; Mihailovic, D.; Arčon, D. A high-temperature quantum spin liquid with polaron spins. Nat. Phys. 2017, 13, 1130-1134. [CrossRef]

17. Shimozawa, M.; Hashimoto, K.; Ueda, A.; Suzuki, Y.; Sugii, K.; Yamada, S.; Imai, Y.; Kobayashi, R.; Itoh, K.; Iguchi, S.; et al. Quantum-disordered state of magnetic and electric dipoles in an organic Mott system. Nat. Commun. 2017, 8, 1821. [CrossRef] [PubMed]

18. Lunkenheimer, P.; Müller, J.; Krohns, S.; Schrettle, F.; Loidl, A.; Hartmann, B.; Rommel, R.; de Souza, M.; Hotta, C.; Schlueter, J.A.; et al. Multiferroicity in an organic charge-transfer salt that is suggestive of electric-dipole-driven magnetism. Nat. Mater. 2012, 11, 755. [CrossRef] [PubMed]

19. Tomić, S.; Pinterić, M.; Ivek, T.; Sedlmeier, K.; Beyer, R.; Wu, D.; Schlueter, J.A.; Schweitzer, D.; Dressel, M. Magnetic ordering and charge dynamics in $\kappa-(\mathrm{BEDT}-\mathrm{TTF})_{2} \mathrm{Cu}\left[\mathrm{N}(\mathrm{CN})_{2}\right] \mathrm{Cl}$. J. Phys. Condens. Matter 2013, 25, 436004. [CrossRef] [PubMed]

20. Abdel-Jawad, M.; Terasaki, I.; Sasaki, T.; Yoneyama, N.; Kobayashi, N.; Uesu, Y.; Hotta, C. Anomalous dielectric response in the dimer Mott insulator $\kappa-(\mathrm{BEDT}-\mathrm{TTF})_{2} \mathrm{Cu}_{2}(\mathrm{CN})_{3}$. Phys. Rev. B 2010, 82, 125119. [CrossRef]

21. Pinterić, M.; Čulo, M.; Milat, O.; Basletić, M.; Korin-Hamzić, B.; Tafra, E.; Hamzić, A.; Ivek, T.; Peterseim, T.; Miyagawa, K.; et al. Anisotropic charge dynamics in the spin-Liquid candidate $\kappa-(\mathrm{BEDT}-\mathrm{TTF})_{2} \mathrm{Cu}_{2}(\mathrm{CN})_{3}$. Phys. Rev. B 2014, 90, 195139. [CrossRef]

22. Tomić, S.; Dressel, M. Ferroelectricity in molecular solids: A review of electrodynamic properties. Rep. Prog. Phys. 2015, 78, 096501. [CrossRef] [PubMed]

23. Pinterić, M.; Lazić, P.; Pustogow, A.; Ivek, T.; Kuveždić, M.; Milat, O.; Gumhalter, B.; Basletić, M.; Čulo, M.; Korin-Hamzić, B.; et al. Anions effects on the electronic structure and electrodynamic properties of the Mott insulator $\kappa$-(BEDT-TTF) ${ }_{2} \mathrm{Ag}_{2}(\mathrm{CN})_{3}$. Phys. Rev. B 2016, 94, 161105(R). [CrossRef]

24. Dressel, M.; Lazić, P.; Pustogow, A.; Zhukova, E.; Gorshunov, B.; Schlueter, J.A.; Milat, O.; Gumhalter, B.; Tomić, S. Lattice vibrations of the charge-transfer salt $\kappa-(B E D T-T T F)_{2} \mathrm{Cu}_{2}(\mathrm{CN})_{3}$ : Comprehensive explanation of the electrodynamic response in a spin-liquid compound. Phys. Rev. B 2016, 93, 081201(R). [CrossRef]

25. Sedlmeier, K.; Elsässer, S.; Neubauer, D.; Beyer, R.; Wu, D.; Ivek, T.; Tomić, S.; Schlueter, J.A.; Dressel, M. Absence of charge order in the dimerized $\kappa$-phase BEDT-TTF salts. Phys. Rev. B 2012, 86, 245103. [CrossRef]

26. Hotta, C. Quantum electric dipoles in spin-liqiod dimer Mott insulator $\kappa-(\mathrm{ET})_{2} \mathrm{Cu}_{2}(\mathrm{CN})_{3}$. Phys. Rev. B 2010, 82, 241104. [CrossRef]

27. Hotta, C. Theories on Frustrated Electrons in Two-Dimensional Organic Solids. Crystals 2012, 2, 1155. [CrossRef]

28. Naka, M.; Ishihara, S. Electronic ferroelectricity in a dimer mott insulator. J. Phys. Soc. Jpn. 2010, $79,063707$. [CrossRef]

29. Naka, M.; Ishihara, S. Collective charge excitation in a dimer Mott insulating system. J. Phys. Soc. Jpn. 2013, 82, 023701. [CrossRef]

30. Naka, M.; Ishihara, S. Quantum melting of magnetic order in an organic dimer-Mott insulating system. Phys. Rev. B 2016, 93, 195114. [CrossRef]

31. Li, H.; Clay, R.T.; Mazumdar, S. The paired-electron crystal in the two-dimensional frustrated quarter-filled band. J. Phys. Condens. Matter 2010, 22, 272201. [CrossRef] [PubMed]

32. Dayal, S.; Clay, R.T.; Li, H.; Mazumdar, S. Paired electron crystal: Order from frustration in the quarter-filled band. Phys. Rev. B 2011, 83, 245106. [CrossRef]

33. Gomi, H.; Ikenaga, M.; Hiragi, Y.; Segawa, D.; Takahashi, A.; Inagaki, T.J.; Aihara, M. Ferroelectric states induced by dimer lattice disorder in dimer Mott insulators. Phys. Rev. B 2013, 87, 195126. [CrossRef]

34. Gomi, H.; Inagaki, T.J.; Takahashi, A. Ferroelectric charge order enhanced by magnetic frustration in dimer Mott insulators. Phys. Rev. B 2016, 93, 035105. [CrossRef]

35. Huse, D.A.; Elser, V. Simple Variational Wave Functions for Two-Dimensional Heisenberg Spin-1/2 Antiferromagnets. Phys. Rev. Lett. 1988, 60, 2351-2354. [CrossRef] [PubMed]

36. Bernu, B.; Lhuillier, C.; Pierre, L. Signature of Néel order in exact spectra of quantum antiferromagnets on finite lattices. Phys. Rev. Lett. 1992, 69, 2590-2593. [CrossRef] [PubMed] 
37. Capriotti, L.; Trumper, A.E.; Sorella, S. Long-range Néel order in the triangular Heisenberg model. Phys. Rev. Lett. 1999, 82, 3899-3902. [CrossRef]

38. Furukawa, T.; Miyagawa, K.; Itou, T.; Ito, M.; Taniguchi, H.; Saito, M.; Iguchi, S.; Sasaki, T.; Kanoda, K. Quantum spin liquid emerging from antiferromagnetic order by introducing disorder. Phys. Rev. Lett. 2015, 115, 077001. [CrossRef] [PubMed]

39. Lazić, P.; Pinterić, M.; Rivas Góngora, D.; Pustogow, A.; Treptow, K.; Ivek, T.; Milat, O.; Gumhalter, B.; Došlić, N.; Dressel, M.; et al. Importance of van der Waals interactions and cation-anion coupling in an organic quantum spin liquid. arXiv 2017, arXiv:1710.01942.

40. Kornelsen, K.; Eldridge, J.E.; Wang, H.H.; Charlier, H.A.; Williams, J.M. Infrared study of the metal-insulator transition in the organic conductor $\kappa-(B E D T-T T F)_{2} \mathrm{Cu}\left[\mathrm{N}(\mathrm{CN})_{2}\right] \mathrm{Cl}$. Solid State Commun. 1992, 4, 343-349. [CrossRef]

41. Williams, J.M.; Kini, A.M.; Wang, H.H.; Carlson, K.D.; Geiser, U.; Montgomery, L.K.; Pyrka, G.J.; Watkins, D.M.; Kommers, J.M.; Boryschuk, S.J.; et al. From Semiconductor-Semiconductor Transition $(42 \mathrm{~K})$ to the Highest- $T_{\mathrm{c}}$ Organic Superconductor, $\kappa-(\mathrm{BEDT}-\mathrm{TTF})_{2} \mathrm{Cu}\left[\mathrm{N}(\mathrm{CN})_{2}\right] \mathrm{Cl}\left(T_{\mathrm{c}}=12.5 \mathrm{~K}\right)$. Inorg. Chem. 1990, 29, 3272-3274. [CrossRef]

42. Sushko, Y.V.; Andres, K. Superconducting Meissner effect under hydrostatic pressure in the ambient-pressure semiconductor $\kappa$-(BEDT-TTF) ${ }_{2} \mathrm{Cu}\left[\mathrm{N}(\mathrm{CN})_{2}\right] \mathrm{Cl}$, where BEDT-TTF is bis(ethylenedithio)tetrathiafulvalene. Phys. Rev. B 1993, 47, 330-333. [CrossRef]

43. Lefebvre, S.; Wzietek, P.; Brown, S.; Bourbonnais, C.; Jérome, D.; Mézière, C.; Fourmigué, M.; Batail, P. Mott Transition, Antiferromagnetism, and Unconventional Superconductivity in Layered Organic Superconductors. Phys. Rev. Lett. 2000, 85, 5420-5423. [CrossRef] [PubMed]

44. Dressel, M.; Gruener, G.; Carlson, K.D.; Wang, H.H.; Williams, J.M. Studies of the Microwave Resistivity of $\kappa-(\mathrm{BEDT}-\mathrm{TTF})_{2} \mathrm{Cu}\left[\mathrm{N}(\mathrm{CN})_{2}\right] \mathrm{Cl}$. Synth. Metals 1995, 70, 927-928. [CrossRef]

45. Sushko, Y.V.; Ito, H.; Ishiguro, T.; Horiuchi, S.; Saito, G. Reentrant superconductivity in $\kappa-(B E D T-T T F){ }_{2} \mathrm{Cu}\left[\mathrm{N}(\mathrm{CN})_{2}\right] \mathrm{Cl}$ and its pressure phase diagram. Solid State Commun. 1993, 87, 997-1000. [CrossRef]

46. Clay, R.T.; Mazumdar, S. From charge- and spin-ordering to superconductivity in the organic charge-transfer solids. arXiv 2018 arXiv:1802.01551.

47. Hiramatsu, T.; Yoshida, Y.; Saito, G.; Otsuka, A.; Yamochi, H.; Maesato, M.; Shimizu, Y.; Ito, H.; Kishida, H. Quantum spin liquid: Design of a quantum spin liquid next to a superconducting state based on a dimer-type ET Mott insulator J. Mater. Chem. C 2015, 3, 1378. [CrossRef]

48. Dressel, M.; Drichko, N. Optical Properties of Two-Dimensional Organic Conductors: Signatures of Charge Ordering and Correlation Effects. Chem. Rev 2004, 104, 5869. [CrossRef] [PubMed]

49. Yamamoto, T.; Uruichi, M.; Yamamoto, K.; Yakushi, K.; Kawamoto, A.; Taniguchi, H. Examination of the Charge-Sensitive Vibrational Modes in Bis(ethylenedithio)tetrathiafulvalene. J. Phys. Chem. B 2005, 109, 15226. [CrossRef] [PubMed]

50. Girlando, A. Charge Sensitive Vibrations and Electron-Molecular Vibration Coupling in Bis(ethylenedithio)tetrathiafulvalene (BEDT-TTF). J. Phys. Chem. C 2011, 115, 19371. [CrossRef]

51. Lang, M.; Lunkenheimer, P.; Müller, J.; Loidl, A.; Hartmann, B.; Hoang, N.H.; Gati, E.; Schubert, H.; Schlueter, J.A. Multiferroicity in the Mott insulating charge-transfer salt $\kappa-(\mathrm{BEDT}-\mathrm{TTF})_{2} \mathrm{Cu}\left[\mathrm{N}(\mathrm{CN})_{2}\right] \mathrm{Cl}$. IEEE Trans. Magn. 2014, 50, 2700107.

52. Kawamoto, A.; Honma, Y.; Kumagai, K.I. Electron localization in the strongly correlated organic system $\kappa-(B E D T-T T F)_{2}$ probed with nuclear magnetic resonance ${ }^{13}$ C-NMR. Phys. Rev. B 2004, 70, 060510(R). [CrossRef]

53. Čulo, M.; Tafra, E.; Basletić, M.; Tomić, S.; Hamzić, A.; Korin-Hamzić, B.; Dressel, M.; Schlueter, J.A. Two-dimensional variable range hopping in the spin-liquid candidate $\kappa$-(BEDT-TTF $)_{2} \mathrm{Cu}_{2}(\mathrm{CN})_{3}$. Phys. B 2015, 460, 208-210. [CrossRef]

54. Nad, F.; Monceau, P.; Kaboub, L.; Fabre, J.M. Divergence of the relaxation time in the vicinity of the ferroelectric charge-ordered phase transition in (TMTTF)2AsF6. Europhys. Lett. 2006, 73, 567-573. [CrossRef]

55. Cava, R.J.; Schneemeyer, L.F.; Fleming, R.M.; Littlewood, P.B.; Rietman, E.A. Effects of impurities on the dielectric response of the charge-density wave in $\mathrm{K}_{0.3} \mathrm{MoO}_{3}$. Phys. Rev. B 1985, 32, 4088-4096. [CrossRef]

56. Ngai, K.L. Relaxation and Diffusion in Complex Systems Springer: New York, NY, USA, 2011; ISBN 978-1-4419-7649-9. 
57. Poirier, M.; Parent, S.; Côté, A.; Miyagawa, K.; Kanoda, K.; Shimizu, Y. Magnetodielectric effects and spin-charge coupling in the spin-liquid candidate $\kappa-(B E D T-T T F)_{2} \mathrm{Cu}_{2}(\mathrm{CN})_{3}$. Phys. Rev. B 2012, 85, 134444. [CrossRef]

58. Manna, R.S.; Hartmann, S.; Gati, E.; Schlueter, J.A.; de Souza, M.; Lang, M. Low-Temperature Lattice Effects in the Spin-Liquid Candidate $\kappa$-(BEDT-TTF $)_{2} \mathrm{Cu}_{2}(\mathrm{CN})_{3}$. Crystals 2018, 8, 87. [CrossRef]

59. Cross, L.E. Relaxor ferroelectrics. Ferroelectrics 1987, 76, 241-267. [CrossRef]

60. Itoh, K.; Itoh, H.; Naka, M.; Saito, S.; Hosako, I.; Yoneyama, N.; Ishihara, S.; Sasaki, T.; Iwai, S. Collective Excitation of an Electric Dipole on a Molecular Dimer in an Organic Dimer-Mott Insulator. Phys. Rev. Lett. 2013, 110, 106401. [CrossRef] [PubMed]

61. Geiser, U.; Wang, H.H.; Carlson, K.D.; Williams, J.M.; Charlier, H.A.; Heindl, J.E.; Yaconi, G.A.; Love, B.J.; Lathrop, M.W.; Schirber, J.E.; et al. Superconductivity at $2.8 \mathrm{~K}$ and $1.5 \mathrm{kbar}$ in $\kappa-(\mathrm{BEDT}-\mathrm{TTF})_{2} \mathrm{Cu}_{2}(\mathrm{CN})_{3}$ : First Organic Superconductor Containing a Polymeric Copper Cyanide Anion. Inorg. Chem. 1991, $30,2586$. [CrossRef]

62. Fukuyama, H.; Kishine, J.; Ogata, M. Energy Landscape of Charge Excitations in the Boundary Region between Dimer-Mott and Charge Ordered States in Molecular Solids. J. Phys. Soc. Jpn. 2017, 86, 123706. [CrossRef]

63. Foury-Leylekian, P.; Ilakovac, V.; Balédent, V.; Fertey, P.; Arakcheeva, A.; Milat, O.; Petermann, D.; Guillier, G.; Miyagawa, K.; Kanoda, K.; et al. $\kappa$-(BEDT-TTF $)_{2} \mathrm{Cu}_{2}(\mathrm{CN})_{3}$ spin liquid: Beyond the average structure. Crystals 2018, 8, 158. [CrossRef]

64. Nakamura, K.; Yoshimoto, Y.; Imada, M. Ab initio two-dimensional multiband low-energy models of EtMe3Sb $\left[\mathrm{Pd}(\mathrm{dmit})_{2}\right]_{2}$ and $\kappa-(\mathrm{BEDT}-\mathrm{TTF})_{2} \mathrm{Cu}_{2}(\mathrm{CN})_{3}$ with comparisons to single-band models. Phys. Rev. $B$ 2012, 86, 205117. [CrossRef]

65. Scriven, E.P.; Powell, B.J. Geometrical Frustration in the Spin Liquid $\beta^{\prime}-\mathrm{EtMe} 3 \mathrm{Sb}\left[\mathrm{Pd}(\mathrm{dmit})_{2}\right]_{2}$ and the Valence-Bond Solid $\mathrm{Me}_{3} \mathrm{EtP}\left[\mathrm{Pd}(\mathrm{dmit})_{2}\right]_{2}$. Phys. Rev. Lett. 2012, 109, 097206. [CrossRef] [PubMed]

66. Tsumuraya, T.; Seo, H.; Tsuchiizu, M.; Kato, R.; Miyazaki, T. Cation Dependence of the Electronic States in Molecular Triangular Lattice System $\beta^{\prime}-\mathrm{X}\left[\mathrm{Pd}(\mathrm{dmit})_{2}\right]_{2}$. J. Phys. Soc. Jpn. 2013, 82, 033709. [CrossRef]

67. Jacko, A.C.; Tocchio, L.F.; Jeschke, H.O.; Valenti, R. Importance of anisotropy in the spin-liquid candidate $\mathrm{EtMe}_{3} \mathrm{Sb}\left[\mathrm{Pd}(\mathrm{dmit})_{2}\right]_{2}$. Phys. Rev. B 2013, 88, 155139. [CrossRef]

68. Alemany, P.; Pouget, J-P.; Canadell, E. Essential role of anions in the charge ordering transition of $\alpha$-(BEDT-TTF) $)_{2} \mathrm{I}_{3}$. Phys. Rev. B 2012, 85, 195118. [CrossRef]

69. Alemany, P.; Pouget, J.-P.; Canadell, E. Electronic structure and anion ordering in (TMTSF) $2 \mathrm{ClO} 4$ and (TMTSF)2NO3: A first-principles study. Phys. Rev. B 2014, 89, 155124. [CrossRef]

70. Alemany, P.; Pouget, J.-P.; Canadell, E. Structural and electronic control of the metal to insulator transition and local orderings in the $\theta$-(BEDT-TTF $)_{2}$ X organic conductors. J. Phys. Condens. Matter 2015, 27, 465702. [CrossRef] [PubMed]

71. Dion, M.; Rydber, H.; Schröder, E.; Langreth, D.C.; Lundqvist, B.I. Van derWaals Density Functional for General Geometries. Phys. Rev. Lett. 2004, 92, 246401. [CrossRef] [PubMed]

72. Román-Pérez G.; Soler, J. M . Efficient Implementation of a van derWaals Density Functional: Application to Double-Wall Carbon Nanotubes. Phys. Rev. Lett. 2009, 103, 096102. [CrossRef] [PubMed]

73. Klimeš, J.; Bowler, D.R.; Michaelides, A. Van der Waals density functionals applied to solids. Phys. Rev. B 2011, 83, 195131. [CrossRef]

74. Mittendorfer, F.; Garhofer, A.; Redinger, J.; Klimeš, J.; Harl, J.; Kresse, G. Graphene on Ni(111): Strong interaction and weak adsorption. Phys. Rev. B 2011, 84, 201401. [CrossRef]

75. Monkhorst, H.J.; Pack, J.D. Special points for Brillonin-zone integrations. Phys. Rev. B 1976, $13,5188$. [CrossRef]

76. Yasin, S.; Dumm, M.; Salameh, B.; Batail, P.; Meziere, C.; Dressel, M. Transport studies at the Mott transition of the two-dimensional organic metal $\kappa-(\mathrm{BEDT}-\mathrm{TTF})_{2} \mathrm{Cu}\left[\mathrm{N}(\mathrm{CN})_{2}\right] \mathrm{Br}_{x} \mathrm{Cl}_{1-x}$. Eur. Phys. J. B 2011, 79, $383-390$. [CrossRef]

77. Sheldrick, G.M. A short history of SHELX. Acta Cryst. A 2008, 64, 112-122. [CrossRef] [PubMed]

(c) 2018 by the authors. Licensee MDPI, Basel, Switzerland. This article is an open access article distributed under the terms and conditions of the Creative Commons Attribution (CC BY) license (http:/ / creativecommons.org/licenses/by/4.0/). 